\title{
Controlling pallidal oscillations in real-time in Parkinson's disease using evoked interference deep brain stimulation (eiDBS): proof of concept in the human
}

\author{
David Escobar Sanabria ${ }^{a}$, Joshua E. Aman ${ }^{a}$, Valentina Zapata Amaya ${ }^{a}$, Luke A. Johnson ${ }^{a}$, Hafsa \\ Farooqi $^{a}$, Jing Wang ${ }^{a}$, Meghan Hill ${ }^{a}$, Remi Patriat ${ }^{b}$, Kelly Sovell-Brown ${ }^{a}$, Gregory F. Molnar ${ }^{a}$, David \\ Darrow $^{c}$, Robert McGovern ${ }^{c}$, Scott E. Cooper ${ }^{a}$, Noam Harel ${ }^{b}$, Colum D. MacKinnon ${ }^{a}$, Michael C. Park ${ }^{c}$, \\ Jerrold L. Vitek ${ }^{\mathrm{a}}$ 而 \\ a Department of Neurology, University of Minnesota, Minneapolis, MN 55455, USA \\ ${ }^{\mathrm{b}}$ Department of Radiology, University of Minnesota, Minneapolis, MN 55455, USA \\ ${ }^{c}$ Department of Neurosurgery, University of Minnesota, Minneapolis, MN 55455, USA \\ ఐCorrespondence: descobar@umn.edu (D.E.S.) and vitek004@umn.edu (J.L.V).
}

\section{Summary}

Approaches to control basal ganglia neural activity in real-time are needed to clarify the causal role of 8-35 Hz ("beta band") oscillatory dynamics in the manifestation of Parkinson's disease (PD) motor signs. Here, we show that resonant beta oscillations evoked by electrical stimulation with precise amplitude and timing can be used to predictably suppress or amplify spontaneous beta band activity in the internal segment of the globus pallidus (GPi) in the human. Using this approach, referred to as closed-loop evoked interference deep brain stimulation (eiDBS), we could suppress or amplify frequency-specific (16-22 Hz) neural activity in a PD patient. Amplification of targeted oscillations led to an increase in the variance of movement tracking delays, supporting the hypothesis that pallidal beta oscillations are linked to motor performance. Our results highlight the utility of eiDBS to characterize the pathophysiology of PD and other brain conditions in the human and develop personalized neuromodulation therapies.

\section{Key Words}

Parkinson's disease, neural oscillations, real-time neural control, closed-loop deep brain stimulation, motor function

\section{Introduction}

While much research has been dedicated to understanding the pathophysiology of Parkinson's disease (PD), the neural circuit dynamics underlying the manifestation of specific motor signs remain to be demonstrated. Current theories propose that the amplitude and incidence of 8-35 Hz "beta" band oscillations, synchronized throughout the basal ganglia thalamocortical (BGTC) circuit, are associated with the severity of motor signs(Brown, 2003; Brown and Williams, 2005; Brown et al., 2001; Kühn et al., 2006, 2009; Little et al., 2012). Although changes in bradykinesia related to levodopa and deep brain stimulation (DBS) treatments have been shown to correlate with the power of local field potential (LFP) activity in the subthalamic nucleus (STN) and internal segment of the globus pallidus (GPi) (Brown, 2003; Brown and Williams, 2005; Brown et al., 2001; Connolly et al., 2015; Escobar Sanabria et al., 2017; Kühn et al., 2006, 2009; Little et al., 2012; Malekmohammadi et al., 2018; Wang et al., 2018), no study has deductively or conclusively demonstrated their causal relationship. DBS yields therapeutic benefit via continuous delivery of high-frequency $(\sim 130 \mathrm{~Hz})$ electrical pulses in the STN or 
GPi. DBS can also suppress beta band oscillations in the target while improving motor function. Yet, the mechanisms by which high-frequency stimulation produces these therapeutic and physiological effects are not clear. This knowledge gap limits our ability to assess the causal relationship between suppression of oscillations attained with DBS and the improvement of motor signs. Does highfrequency stimulation directly reduce beta band oscillations and thereby produce a therapeutic effect? Or is the reduction in beta band oscillations during DBS secondary (or unrelated) to the therapeutic effects of DBS? Previous studies have attempted to answer these questions using $20 \mathrm{~Hz}$ electrical stimulation of the STN, with the idea that stimulation at this frequency may promote the generation of STN rhythms in the beta band. While studies have reported that $20 \mathrm{~Hz}$ STN stimulation can worsen bradykinesia in some PD patients(Chen et al., 2007; Eusebio et al., 2008; Fogelson et al., 2005), others have challenged this idea and have shown no effect(Blumenfeld et al., 2015; Huang et al., 2014). Supporting the latter argument, a recent study showed that open-loop stimulation of the STN of PD patients, delivered at the same frequency as the STN beta oscillations, on average did not amplify these beta oscillations(Holt et al., 2019). The above studies highlight the need for approaches that can control beta band oscillations in real-time, without employing high-frequency stimulation, to characterize the role of these oscillations in the manifestation of motor signs in PD.

In the current study, we demonstrate that resonant oscillations in the human GPi evoked by stimulation pulses in the GPi can be employed to suppress or amplify frequency-specific spontaneous GPi oscillations in real-time without utilizing high-frequency stimulation. We used a feedback (closed-loop) control strategy in which stimulation pulses were delivered in the GPi with precise amplitude and timing relative to the targeted GPi oscillations to evoke neural responses that suppress or amplify these oscillations. The rationale behind this approach, referred to as closed-loop evoked interference DBS (eiDBS), is that synaptic-related neural responses evoked by electrical pulses can "override" spontaneous, synaptic-related oscillations via synaptic integration when the pulses are delivered with precise amplitude and timing relative to the phase of spontaneous oscillatory activity (Escobar Sanabria et al., 2020). See schematic of eiDBS in Fig. 1A. eiDBS is suitable to characterize the causal (direct or indirect) link between neural oscillations and motor function because it can suppress or amplify frequency-specific neural activity in a predictable manner without utilizing high-frequency isochronal pulses.

We tested eiDBS in a PD patient implanted with a directional DBS lead in the GPi and evaluated the effect of beta band suppression and amplification on movement tracking performance. eiDBS was capable of suppressing or amplifying GPi oscillations in the targeted frequency band $(16-22 \mathrm{~Hz})$ in realtime. Stimulation-evoked responses (ERs) that mediated this modulation resonated in the beta band, within the same frequency range where the peak power of the spontaneous LFPs was located. Because the ERs resided in the beta band, eiDBS required less stimulation amplitude to modulate beta oscillations than the stimulation needed to modulate neural activity in other frequency bands.

Amplification of targeted oscillations attained via eiDBS was associated with an increase in the variance of movement tracking delays in a rapid alternating movement (RAM) task, supporting the idea of a link (direct or indirect) between controlled changes in pallidal beta oscillations and motor performance. Suppression of targeted oscillations did not have a significant effect on RAM performance in this patient, likely due to a floor effect associated with the mild UPDRS-III bradykinesia subscores in the offstimulation condition. This study highlights the utility of eiDBS as a tool to characterize the pathophysiology of PD and other brain conditions in the human and the prospect of developing personalized neuromodulation treatments based on interference between resonant stimulation-evoked and spontaneous neural activity. 


\section{Results}

LFP and evoked response oscillations matched frequency and location. We characterized spontaneous and stimulation-evoked neural activity and evaluated the feasibility of delivering eiDBS in a PD patient implanted with a directional DBS lead in the sensorimotor region of the GPi (Fig. 1B). See recording and stimulation approach in the Methods Section. Resting state, off-stimulation LFPs recorded from the patient's GPi exhibited elevated beta band oscillations with a peak frequency of 19 $\mathrm{Hz}$ in the power spectral density (PSD). See Fig. 1C. We selected the frequency band between 16 and $22 \mathrm{~Hz}$ for modulation via eiDBS. Open-loop, low-frequency electrical stimulation pulses $(2.93 \mathrm{~Hz}, 60 \mathrm{us}$ pulse width) evoked neural responses with highest power at $20.8 \mathrm{~Hz}$, near the peak frequency of the spontaneous LFP PSD (i.e., $19 \mathrm{~Hz}$ ). See Figs. 1C,E. The amplitude of the GPi ERs was insignificant for stimulation currents equal to $0.5 \mathrm{~mA}$ but clear and significant for currents equal to 2 and $3 \mathrm{~mA}$. This nonlinear response to stimulation is a characteristic of neural stimulation-evoked responses(Escobar Sanabria et al., 2020).

For eiDBS to have a true modulatory effect on spontaneous neural activity, the ERs measured with the DBS lead need to be generated by the same neuronal population that give rise to the LFP spontaneous oscillations. We used the spatial distribution of electric potentials across differential recordings from the directional DBS lead to evaluate whether the ER and LFP spontaneous oscillations were generated by the same neural source. Across all available differential potentials from the directional DBS lead, the amplitude of the ERs was highly correlated with the amplitude of the spontaneous beta oscillations (Correlation coefficient $\mathrm{R}=0.8$, slope $=1.37, \mathrm{p}=0.028$ ). See Fig. 1F. This high correlation is a necessary condition for the neural sources (monopolar or dipolar) generating the ER and spontaneous oscillations to be in the same location according to the Poisson equation of electrostatics(Griffiths, 2017; Nunez and Srinivasan). The solution to this equation implies that the spatial distribution of electric potentials generated by two neural sources should be the same if these neural sources are at the same location.

eiDBS was capable of suppressing or amplifying frequency-specific GPi activity. We constructed mathematical models of the ERs based upon the patient's data using system identification techniques (Fig. 2A,B) as described in more detail in the Methods Section and preclinical work published previously(Escobar Sanabria et al., 2020). These ER models are described by linear differential equations and a saturation element that transforms the biphasic stimulation pulses to monophasic pulses responsible for the neural response. Using the patient-specific ER mathematical models and recorded LFP data, we constructed a computer simulation (Fig. 2A,B) to characterize the neural modulation attained with eiDBS and search for the stimulation parameters (amplitude and phase angle) that maximized suppression and amplification of neural activity in the targeted band (16-22 Hz). See optimization curve in Fig. 2C and computer simulation with optimized parameters in Fig. 2D. The parameter search was performed with stimulation amplitudes for which ER and stimulation-artifact models were available from the recorded data $(0.5,2.0$, and $3.0 \mathrm{~mA})$. The computer simulation (Fig. 2D) indicated that when neural activity was suppressed in the targeted frequency band, there was amplification of activity in adjacent frequencies $(\sim 15 \mathrm{~Hz}$ and $\sim 23 \mathrm{~Hz}$ ). The side-band amplification effect occurs due to phase distortions introduced by the filter at frequencies adjacent to the targeted band, and given that optimal stimulation parameters for one frequency are not optimal for another.

We tested eiDBS in-vivo with the study participant using the stimulation parameters found to be optimal (stimulation at $2 \mathrm{~mA}$ and phase angles equal to -85 and 95 deg for suppression and amplification). eiDBS was capable of suppressing or amplifying pallidal activity in the targeted frequency interval (16$22 \mathrm{~Hz}$ ). See Figs. 3A-D. eiDBS (suppression or amplification) was well tolerated by the patient. During 
the suppression stage of the eiDBS experiment, the median LFP amplitude in the targeted band decreased from 4.59 to $2.74 \mathrm{uV}(\mathrm{p}=6.12 \mathrm{e}-08$ with rank-sum test, Cohen's U3 effect size=1). During the amplification stage, the median amplitude of oscillations in the targeted band increased from 4.59 to $7.25 \mathrm{uV}(\mathrm{p}=4.04 \mathrm{e}-8$ with rank-sum test, Cohen's U3 effect size $=1)$. Suppression of neural activity in the targeted band resulted in an increase in the median amplitude of the LFP in the $12-16 \mathrm{~Hz}$ band (3.63 $\mathrm{uV}$ in the off stimulation vs. $5.01 \mathrm{uV}$ in the suppression condition, $\mathrm{p}=4.5 \mathrm{e}-7$ with rank-sum test, Cohen's U3 effect size $=1$ ). While an increase in power at $\sim 23 \mathrm{~Hz}$ is observed in the suppression condition (spectrogram of Fig 3A), our statistical analysis indicated that no significant changes occurred in the 22$26 \mathrm{~Hz}$ band. See the Methods Section for a description of the statistical analysis approach.

Stimulation-evoked responses in the GPi mediated the modulation achieved via eiDBS. The computer simulations constructed with the ER mathematical models and patient-specific LFP activity (Fig. 2D) predicted the modulatory effects of eiDBS in the suppression and amplification conditions during the in-vivo experiments with the subject. The ability of the computer simulation to predict the modulatory effect of eiDBS indicates that the ERs mediated the suppression or amplification of spontaneous oscillations in the patient, given that the ER mathematical model drove the computer simulations. Moreover, the ER transfer function (input-output dynamic map) indicates that the highest gain of this transfer function is at $19.9 \mathrm{~Hz}$. See Methods Section. Therefore, periodic stimulation at 19.9 $\mathrm{Hz}$ yields evoked responses larger than stimulation at any other frequency. When these evoked responses have the same amplitude and are out of phase with spontaneous oscillations at the same frequency (i.e., eiDBS), a maximum suppression of these spontaneous oscillations can be achieved. This analysis suggests that eiDBS in this patient could suppress or amplify spontaneous beta activity in the targeted band $(16-22 \mathrm{~Hz})$ with minimum stimulation current as compared with oscillations at other frequency bands.

\section{Modulation of targeted oscillations was associated with changes in the variance of rapid alternating movement tracking. We quantified the effect of eiDBS on movement tracking} performance via a rapid alternating movement (RAM) task in which the subject rotated the forearm (pronation/supination) following a periodic auditory cue (1 beep/s). We used the time delay between beep onset and the peak angular displacement of a movement cycle to measure tracking performance. See assessment system and schematic of movement task in Figs. 4A,B. We evaluated how the variance of movement tracking delays changed between conditions (e.g., off-stimulation vs. eiDBSamplification) by comparing the dispersion of the time delay distributions via the Square Rank Test. See Fig. 4C,D. The median absolute deviation (MAD) was employed as a nonparametric scalar measurement of time delay variance in each condition (Fig. 4C). Amplification of $16-22 \mathrm{~Hz}$ oscillations attained with eiDBS (eiDBS-amplification condition) was associated with an increase in the variance of RAM tracking delays as compared to the off-stimulation condition $(p=0.036$, Square Rank Test corrected for two comparisons via Bonferroni method). See Fig. 4D. Suppression of the targeted neural oscillations (eiDBS-suppression condition) did not have a significant effect on the tracking delay variance as compared to the off-stimulation condition. This result may reflect a floor effect associated with the mild UPDRS bradykinesia subscores obtained two hours before the experiments and before DBS lead placement. The UPDRS-III bradykinesia subscores associated with pronation supination and upper arm movement were equal to 0/4 and 0/4 two hours before eiDBS testing, and 0/4 and 1/4 eight months before the eiDBS experiments during the most recent clinic visit in which off-medication assessments were performed.

\section{Discussion}


Significance and related work. We previously developed the concept of eiDBS using the 1-methyl-4phenyl-1,2,3,6-tetrahydropyridine (MPTP) nonhuman primate model of PD and showed that amplification or suppression of STN oscillations could be achieved using STN neural responses evoked by stimulation in the GPi(Escobar Sanabria et al., 2020). Here, we demonstrate the feasibility of controlling beta band oscillations in the human GPi in real-time by using resonant neural responses evoked by stimulation of the GPi. This approach (eiDBS) is suitable to characterize the causal (direct or indirect) link between oscillations and motor function because suppression or amplification of neural activity within a selected frequency band is attainable in a predictable manner without employing highfrequency isochronal pulses. eiDBS modulates beta oscillations with and stimulation frequency that in average is equal to the mean frequency of the targeted oscillations. Not using high-frequency stimulation is critical to characterizing the role of beta band oscillations in PD, given that high-frequency pulses can improve motor function and also suppress beta band activity. However, it is unclear whether high-frequency stimulation directly reduces beta band oscillations and thereby produces a therapeutic effect, or the reduction in beta band oscillations is unrelated to the therapeutic effect of high-frequency stimulation.

Leveraging the sensing capabilities of directional DBS leads, we showed that the neural sources generating both spontaneous and stimulation-evoked oscillations are likely the same, indicating that 1) eiDBS attains true neural modulation, and 2) observed modulation is not the effect of volume conduction from neural sources located in distinct regions. Furthermore, we showed that patientspecific ER mathematical models combined with LFP recordings can be used to predict the stimulation parameters that maximize the suppression or amplification of spontaneous, frequency-specific neural activity in the human GPi. Therefore, eiDBS can be programmed based on neurophysiological data that is specific to the particular patient. Our data from the studied patient also suggest that controlled changes in beta band activity can alter motor tracking performance, supporting the hypothesis that beta band oscillatory dynamics are causally (directly or indirectly) linked to motor dysfunction in PD.

Our results inform future studies directed at investigating the causal role of frequency- and locationspecific neural activity in the manifestation of specific PD motor and non-motor signs using eiDBS. They are also a step towards developing closed-loop DBS therapies that control circuit-wide neurophysiological dynamics underlying the generation of neural oscillations and brain dysfunction in real-time. While we delivered electrical stimulation with precise amplitude and timing (phase), one should note that the rationale behind using phase feedback is different from other approaches intended to induce phase desynchronization across neurons(Holt et al., 2016; Tass, 2002), modulate depolarization or hyperpolarization of neurons in the proximity of the stimulation site(Peles et al., 2020), alter short-term plasticity(Zanos et al., 2018), or deliver stimulation based on kinematic variables as tremor(Cagnan et al., 2017) or gait(Louie et al., 2021). eiDBS continuously overrides the inputs (synaptic-related) of a targeted neuronal population to suppress or amplify spontaneous oscillations via interference created with neural responses evoked by electrical stimulation (low-frequency, synaptic related). Our current implementation of eiDBS with a fixed stimulation phase and amplitude can be further optimized to account for dynamic changes in the amplitude and frequency of neural oscillations and thereby maximize the suppression of neural activity across frequencies in real-time. Future realtime optimization routines require neurostimulators that enable instantaneous changes in parameters and advanced multi-objective feedback control algorithms.

Role of stimulation evoked responses in neural control. Modulation of spontaneous oscillations in the GPi achieved by eiDBS was mediated by neural oscillatory activity evoked by stimulation within the GPi. The pallidal evoked responses resonated at the same frequency (within the beta band) where the 
spontaneous oscillations resided, suggesting that intrinsic resonant properties of circuits connected to the GPi, thought to play a role in the generation of spontaneous beta band oscillations in PD (Eusebio et al., 2009), may also underlie the generation of evoked responses. This resonance in the beta band enabled eiDBS to modulate beta band oscillations with minimal stimulation amplitude as compared with other frequency bands. This reduced stimulation amplitude (and energy) is a critical feature for stimulation therapies to minimize possible side effects associated with unwanted activation of neuronal pathways and for implantable devices to minimize battery replacements or recharging frequency.

Although the circuit-wide mechanisms underlying the generation of either spontaneous or stimulationevoked beta band oscillations in the GPi of PD patients are unknown, these oscillations are likely associated with the activation of multi-synaptic feedback loops in the basal ganglia-thalamocortical network(Bevan et al., 2002; Johnson and McIntyre, 2008; Wilson and Bevan, 2011). For eiDBS to result in true neural modulation, the ER and spontaneous oscillatory activity need to be generated by the same population of interconnected neurons. The exact location of neural sources within the GPi generating the spontaneous or stimulation-evoked oscillations measured with the directional DBS lead is challenging to estimate using the limited number of LFP channels (i.e., eight) from the lead, restricted spatial coverage of the lead contacts, and uncertain electrical properties of the tissue surrounding the lead contacts. Nevertheless, the tight correlation between the amplitude of spontaneous and stimulation-evoked oscillations across LFP montages created with the directional DBS lead is evidence of these oscillations being generated at the same location and not associated with volume conduction of independent sources at distinct sites. The rationale behind this argument is that two current sources (dipolar or monopolar) located within the same regions in a volume conductor generate the same electric potential profile in space (Poisson equation of electrostatics)(Griffiths, 2017; Nunez and Srinivasan).

Limitations. The data presented in this study is from a single subject, and results cannot be generalized until a large cohort of patients is studied. Nevertheless, this study considered multiple samples of behavioral and electrophysiological data that allowed us to perform a well-powered statistical analysis within a subject. A limitation of the eiDBS algorithm implemented here is that suppression of oscillations in the targeted frequency band resulted in amplification of neural activity in an adjacent band (12-16 Hz). This side-band amplification can be a confounding factor when analyzing the effect of suppressing targeted neural activity via eiDBS on behavior. For example, in our experiments, the side-band amplification could influence the movement tracking performance during the suppression phase of the experiments. Future eiDBS systems need to track the oscillations' frequency and reduce filtering-related phase distortions to minimize side-band amplification during the suppression of neural activity. We also acknowledge that our analysis of motor performance could be influenced by the patient's fatigue as the eiDBS-amplification condition was tested after the offstimulation and eiDBS-suppression conditions. Nevertheless, we allowed the patient to rest for at least 20 s between condition transitions to minimize the effect of fatigue on movement performance.

\section{Acknowledgments}

Research reported in this publication was funded by the Wallin Discovery Fund, the Engdahl Family Foundation, the Kurt B. Seydow Dystonia Foundation, the National Institute of Neurological Disorders and Stroke (P50-NS098573, R01-NS037019), and the University of Minnesota's MnDRIVE (Minnesota's Discovery, Research and Innovation Economy) Initiative. We thank Kevin Patino Sosa for 
building lead adapters and connectors, and Stephanie Alberico and Kevin O'Neill for help during data collection.

\section{CRediT Authorship Contributions}

David Escobar Sanabria: Conceptualization, Methodology, Software, Validation, Formal Analysis, Investigation, Resources, Data Curation, Writing - Original Draft, Visualization, Supervision, Project Administration, Funding Acquisition. Joshua E. Aman: Resources, Investigation, Data Curation, Supervision, Project Administration. Valentina Zapata: Software, Formal Analysis, Data Curation, Visualization. Luke A. Johnson: Software, Investigation, Visualization, Writing - Review \& Editing. Hafsa Farooqi: Software, Data Curation, Visualization. Jing Wang: Investigation, Writing - Review \& Editing. Meghan Hill: Investigation, Writing - Review \& Editing. Remi Patriat: Data Curation, Writing Review \& Editing. Kelly Brown: Project Administration. Gregory F. Molnar: Project Administration, Funding Acquisition. David Darrow: Investigation, Resources. Robert McGovern: Investigation, Resources, Writing - Review \& Editing. Scott E. Cooper: Resources, Project Administration, Writing Review \& Editing Funding Acquisition. Noam Harel: Resources, Funding Acquisition. Colum D. MacKinnon: Methodology, Resources, Supervision, Funding Acquisition. Michael C. Park: Methodology, Investigation, Resources, Supervision, Funding Acquisition. Jerrold L. Vitek: Conceptualization, Resources, Supervision, Writing - Review \& Editing, Funding Acquisition, Project administration.

\section{Declaration of Interests}

R. Patriat is a consultant for Surgical Information Sciences Inc. G.F. Molnar has previously consulted for Abbott. N. Harel is a shareholder of Surgical Information Sciences. M.C. Park is listed faculty for University of Minnesota Educational Partnership with Medtronic, Inc. and has been a consultant for Zimmer Biomet, Synerfuse, Inc., NeuroOne Medical Technologies Corp., Boston Scientific, and Surgical Information Sciences, Inc. J. L. Vitek has served as a consultant for Medtronic, Boston Scientific, and Abbott and serves on the scientific advisory board for Surgical Information Sciences.

\section{Figure Legends}

Figure 1. (A) Schematic of closed-loop evoked interference DBS. eiDBS delivers stimulation pulses with precise amplitude and timing to evoke resonant neural responses that overrides spontaneous oscillations via constructive or destructive interference. (B) Diagram of directional DBS lead and localization of directional contacts in the GPi adapted from (Johnson et al.). The 2D slice in the axial plane is at the depth of ring 3 in the DBS lead. The orthogonal coordinate frame depicted on the axial plane consists of the anterior $(A)$-posterior $(P)$ and medial $(M)$-lateral $(L)$ axes. $(C)$ Power spectral density (PSD) of LFPs recorded from contacts 2c-4. (D) Neural responses in the GPi evoked by stimulation in the GPi with currents equal to 0.5, 2.0, and 3.0 mA. (E) Wavelet transform scalogram (time-frequency map) of GPi response evoked by $3 \mathrm{~mA}$ stimulation pulses. Regions where the ER is not significantly greater in amplitude than surrogate data are depicted in gray, predominately in the upper right and left hand corners of the scalorgram. Colored regions (not gray) correspond to regions where the ER is significantly greater than surrogate data $(p<0.01)$. (F) Scatter plot of ER amplitude vs. spontaneous activity amplitude in the $16-22 \mathrm{~Hz}$ band as observed across differential recordings from 
the DBS directional lead. Scalar measures of ER amplitude for each montage are equal to the sum of scalogram values over frequencies in the targeted band $(16-22 \mathrm{~Hz})$ at the time where the maximum ER amplitude is observed. Scalar measures of the spontaneous LFP amplitude are equal to the sum of amplitude spectral density (square root of PSD) values over frequencies in the targeted band. $R=0.8$ is the correlation coefficient associated with the ER and spontaneous activity data points.

Figure 2. (A) Schematic of eiDBS computer simulation in which stimulation pulses are triggered by a closed-loop controller based on real-time LFP measurements. The model consists of a static saturation nonlinearity that allows us to capture the symmetric response of the GPi to cathodal and anodal stimulation pulses. The negative phase of the stimulus is the input of a linear time-invariant system of differential equations that reproduce the evoked response temporal dynamics. The LFP measurement is modeled as the linear superposition of the ER and spontaneous oscillations. (B) Measurement and mathematical model of GPi ER for a stimulation amplitude of 2mA. (C) Effect of eiDBS (2 mA) delivered at different phase angles on the mean amplitude of targeted neural activity in the $16-22 \mathrm{~Hz}$ band (computed over $60 \mathrm{~s}$ ). The curve indicates that the optimal phase angles for suppressing and amplifying the targeted neural activity are -85 and 95 deg, respectively. (D) Spectrogram (LFP power in the time-frequency domain) of computer simulation in which eiDBS was delivered with parameters found to maximize the suppression and amplification of $16-22 \mathrm{~Hz}$ oscillations.

Figure 3. (A) Spectrogram (LFP power in the time-frequency domain) of LFP activity during periods in which eiDBS was delivered to suppress and amplify targeted GPi oscillations in a PD patient implanted with a directional DBS lead. (B) Temporal dynamics of modulated oscillations (filtered in the 17-21 Hz range for clear visualization of modulatory effects on $\sim 19 \mathrm{~Hz}$ oscillations) in the off-stimulation to suppression, and suppression to amplification transitions. (C) Mean power spectral density (PSD) of LFP activity in the off-stimulation, suppression, and amplification conditions illustrates how eiDBS modulated spontaneous pallidal oscillations across frequencies. (D) Boxplot with independent measurements (mean over $3 \mathrm{~s}$ ) of the oscillations' amplitude envelope in the targeted band (16-22 Hz) in the off-stimulation, eiDBS-suppression, and eiDBS-amplification conditions. The box edges represent the interquartile range, and the horizontal line within each box represents the median. Most extreme data points not considered outliers are represented by the whiskers. The amplitude of oscillations significantly decreased from the off-stimulation to the suppression condition $(p=6.12 e-08$, Cohen's U3 effect size $=1$ ) and increased from the off-stimulation to the amplification condition ( $p=4.04 e-8$, Cohen's U3 effect size=1). P-values were corrected for the two comparisons made using the Bonferroni method. The symbol 0 indicates that the difference between conditions was statistically significant with the $p$ values listed above. The number of independent observations (mean amplitude over $3 \mathrm{~s}$ ) used in this analysis was $n=16$ in the off-stimulation, $n=37$ in the suppression, and $n=29$ in the amplification condition.

Figure 4. (A) Manipulandum system used to assess movement tracking performance. (B) Schematic of rapid alternating movement (RAM) task used to assess tracking performance. Auditory cues ("beeps") were generated to guide the patient's forearm motion in the clockwise $(R)$ or counterclockwise direction (L). The time delays (e.g., $\Delta t_{1}$ and $\Delta t_{2}$ ) between the onset of the beep and peak angular position of the forearm were used to characterize tracking performance. (C) Median absolute deviation (MAD) of time delay measurements in the off-stimulation, suppression, and amplification conditions from experimental data. MAD is a nonparametric measurement of variance. (D) Distributions of time delays in the offstimulation (left), suppression (middle), and amplification (right) conditions. The Square Rank Test indicates that the variance in the distribution of time delays in the amplification condition is significantly 
greater than the distribution in the off-condition $(p<0.036$, corrected for two comparisons with the Bonferroni method).

\section{Methods}

Patient and surgical procedure. All patient procedures were approved by the University of Minnesota Institutional Review Board (IRB protocol \#1701M04144) with consent obtained according to the Declaration of Helsinki. This study was conducted with a male patient ( 55 years old) diagnosed with idiopathic PD 6 years before unilateral (right side) GPi DBS surgery. Intraoperative microelectrode mapping was used to identify the sensorimotor region of GPi for DBS lead placement(DeLong, 1971; Elder et al., 2005; Georgopoulos et al., 1983; Vitek et al., 1998). Following intraoperative microelectrode mapping, a directional DBS lead (Boston Scientific Vercise Cartesia model DB-2202-45; $1.5 \mathrm{~mm}$ contact height with $0.5 \mathrm{~mm}$ vertical spacing) was implanted. See diagram of the DBS lead in Fig. 1B. After DBS implantation, a lead extension was tunneled to a subcutaneous pocket in the chest and connected to another extension that was externalized through an abdominal incision(Aman et al., 2020). Five days later, the patient was admitted at the University of Minnesota Clinical Research Unit, where experiments took place over the course of two days.

Electrode localization. The location of the DBS lead contacts in the GPi was confirmed based on information obtained during intraoperative electrophysiological mapping as well as co-registered preoperative 3T MRI and postoperative CT scans(Aman et al., 2020). Electrode localization and orientation for this patient was previously described in (Johnson et al.). Briefly, the lead orientation relative to the brain was derived using a modified version of the DiODe algorithm(Hellerbach et al., 2018) and based on unique artifacts of the lead contacts and fiducial marker superior to the most distal contact from the lead tip. The orientation of the lead was confirmed with information extracted from fluoroscopy and X-ray images acquired intraoperatively.

Postoperative externalized recordings. The externalized lead extension was connected to an ATLAS neurophysiological recording system (Neuralynx, Bozeman, MT, USA) via customized connectors. Reference and ground EEG electrodes were placed on the scalp along the midline. An FDA-approved neurostimulator (g.Estim, g.tec, Schiedlberg, Austria) was employed to deliver current-controlled stimulation. A skin-surface patch electrode was placed on the chest contralateral to the DBS lead to serve as a current return for monopolar electrical stimulation. On day 1 of the externalization recordings, we collected resting LFP data in the off-stimulation condition and during low-frequency ( 3 $\mathrm{Hz}$ ), monopolar stimulation $(0.5,2$, and $3 \mathrm{~mA}$ with 60 us pulse width). These data were used to characterize evoked responses and calculate eiDBS parameters that maximize suppression or amplification of beta band oscillations. See the Optimization of eiDBS Section. In the morning of day 2, we tested eiDBS with the parameters found on day 1 in the off-medication state, 16 hours after the last dose of carbidopa/levodopa (1 pill, 25/100 mg). Rapid alternating movement (RAM) tracking performance was assessed in the following order: off-stimulation, suppression, and amplification condition. For each condition, we tested four sequences of the RAM task as described below.

Characterization of stimulation-evoked responses. All data analyses were performed using custom software developed in MATLAB (The MathWorks, Natick, MA). Evoked responses in the GPi were computed by averaging LFP segments aligned with stimulation pulses. Stimulation artifacts were removed as described below in the eiDBS implementation Section. The amplitude of evoked responses in the frequency and time domain were characterized using scalograms (time-frequency maps) calculated with the wavelet transform. We assessed whether observed evoked responses in the 
scalograms were the result of chance using a permutation test without replacement, performed by randomly sampling 10,000 resting state LFP segments and computing scalograms for each permutation. By using the permutation distribution of surrogate scalograms, we computed the $p$-value of the original wavelet value at each frequency and time. A false discovery rate (FDR) correction for multiple tests in the time and frequency domains was applied to the p-values. Values of wavelet amplitudes with corrected $p<0.01$ were considered significant.

The effect of stimulation pulses on the ER temporal dynamics was characterized using a saturation nonlinearity (static) connected to a system of linear differential equations (dynamics) as depicted in Fig. 2A. This model has been shown to estimate the response of ER to stimulation pulses accurately(Escobar Sanabria et al., 2020). The parameters of the differential equations were obtained using the instrumental variable system identification approach(Ljung, 1986). A transfer function with four poles and one zero was selected using Akaike's Information Criterion (AIC) to minimize both the model prediction error and the number of estimated parameters(Ljung, 1986). The following differential equations describe the continuous-time input-output model:

$$
\begin{gathered}
\dot{x}=A x+B u \\
y=C x,
\end{gathered}
$$

where $x$ is the state vector, $\dot{x}$ is the derivative of $x$ with respect to time, $u$ is the saturated input stimuli (uA), $y$ is the ER output (uV), and $A, B$, and $C$ are constant matrices that parameterize the differential equations. These matrices are given as follows.

$$
\begin{gathered}
A=\left[\begin{array}{ccccc}
-105.4 & -223.7 & -119.2 & -81.62 & -42.25 \\
128 & 0 & 0 & 0 & 0 \\
0 & 128 & 0 & 0 & 0 \\
0 & 0 & 128 & 0 & 0 \\
0 & 0 & 0 & 64 & 0
\end{array}\right] \\
B=\left[\begin{array}{l}
8 \\
0 \\
0 \\
0 \\
0
\end{array}\right] \\
C=\left[\begin{array}{lllll}
0 & 0 & 0 & -4.835 & 1.013
\end{array}\right]
\end{gathered}
$$

The largest gain of the input-output transfer function described by the above linear dynamics is at 19.9 $\mathrm{Hz}$ (largest gain in the Bode magnitude diagram). Therefore, periodic stimuli at this frequency leads to the highest amplitude evoked responses, implying that a minimum stimulation amplitude is needed to suppress or amplify oscillations at $19.9 \mathrm{~Hz}$ as compared to oscillations at other frequencies.

Oscillatory activity. Artifacts were removed from the LFP raw data as described below in the Implementation of eiDBS Section, and then the LFPs were down-sampled at $3 \mathrm{KHz}$ for processing. The power of the LFP recordings in the time and frequency domain was characterized using spectrograms and the Welch method. We measured the average power in a specific condition (e.g., eiDBS suppression) using PSD curves computed with the Welch method. We assessed whether the amplitude of neural oscillations in the targeted frequency band changed when eiDBS was delivered by using scalar measurements of the oscillations' amplitude envelope. These scalar measurements were computed by filtering the artifact-suppressed data in the targeted band, calculating the magnitude of the analytic signal via the Hilbert transform, and averaging the amplitude envelope over non-overlapping windows of three-second duration and separated by one second. This separation time is greater than 
the maximum time between effectively independent data, calculated across conditions (off stimulation, amplification, suppression) by using the autocorrelation function(Escobar Sanabria et al., 2020; Guemas et al., 2014, 2011). Pairwise differences between scalar measurements of the oscillations' amplitude in two different conditions were assessed via the Wilcoxon rank-sum test. The p-values resulting from this test were corrected for the two comparisons via the Bonferroni method. We assumed that the difference between measurements in the two conditions was significant when $p<0.05$. We evaluated effect sizes using the Cohen signed (non-parametric) test ('U3')(Hentschke and Stüttgen, 2011).

Real-time neural control infrastructure. An industrial computer dedicated to modulating brain activity in real-time with a closed-loop delay of less than $1 \mathrm{~ms}$ was used to implement eiDBS (Mobile Target Machine, SpeedGoat, Bern Switzerland). The real-time computer was connected to the ATLAS recording system via a fiber optic link (Gigabit Ethernet) to read LFP data at $24 \mathrm{KSamples/s}$ and to a g.Estim stimulator via a digital interface to control individual stimulation pulses.

Implementation of eiDBS. The algorithm used to implement eiDBS has been previously described in preclinical studies with nonhuman primates(Escobar Sanabria et al., 2020). Briefly, this algorithm consists of 1) acquiring LFP data from the ATLAS system, 2) suppressing stimulation artifacts using a dynamic template model of the artifact and blanking residual artifacts (2.6 ms blanking duration), 3 ) filtering differential LFPs in the targeted frequency band, and down-sampling LFPs at $3 \mathrm{KHz}, 4$ ) computing the instantaneous phase and amplitude of the filtered LFP using a Hilbert transformer filter, and 5) triggering single stimulation pulses at specific phases of the neural oscillations. Stimulation was not delivered if the amplitude envelope of these oscillations was less than a prescribed threshold. This threshold was equal to the 20th percentile of the oscillations' amplitude envelope, calculated in the offstimulation condition.

For sensing, we selected contacts $2 \mathrm{c}$ and 4 , whose differential LFP had the largest signal-to-noise ratio in the frequency band targeted for modulation (16-22 Hz). The sensing montage was selected among the following combinations: 1-3a, 1-3b, 1-3c, 2a-4, 2b-4, and 2c-4. We assessed these electrode combinations to enable monopolar stimulation to be delivered through the ring located between sensing electrodes and thereby minimize stimulation artifacts via differentiation of the sensing electrodes. Stimulation was delivered using monopolar stimulation through ring $3 a b c$ segments $3 a$, $3 b$, and $3 c$ tied together). We targeted a frequency band centered on the peak of the LFP power spectral density. LFPs were filtered using a second-order Butterworth filter with a $6 \mathrm{~Hz}$ bandwidth.

Optimization of eiDBS. A computer optimization (search) for eiDBS was performed based on the ER mathematical models described above to determine the stimulation parameters that maximize suppression of neural oscillations in the targeted band(Escobar Sanabria et al., 2020). We created patient-specific computer simulations using these evoked response models and simulated the closedloop algorithms with a range of stimulation phase angles for a given stimulation amplitude. A search across phase angles (-180 to $175 \mathrm{deg}$ with a 5-deg. resolution) for a stimulation current of $2 \mathrm{~mA}$ was performed to calculate the phase that minimized the amplitude of neural activity in the targeted frequency band. The same stimulation amplitude determined for eiDBS-suppression was also used for eiDBS-amplification. The stimulation phase for eiDBS-amplification was determined using the search described above with the given stimulation amplitude.

Quantification of rapid alternating movement (RAM). Quantitative measures of RAM were obtained using a custom-built robotic manipulandum (Fig. 4A, Entact Robotics, Toronto, Canada). The patient was instructed to actively rotate the handle back and forth in sync with a rhythmic auditory tone cue (beep), with the desired range of motion $\left( \pm 15^{\circ}\right)$ and real-time visual feedback of position provided on a monitor. Auditory cues were given with a rate of $1 \mathrm{beep} / \mathrm{s}$. The patient's movement performance was 
assessed in each condition (e.g., eiDBS-amplification) with four sequences of 20 movement cycles. Sequences were separated by a rest period of at least $20 \mathrm{~s}$. We tested the off-stimulation condition first, followed by the eiDBS-suppression and eiDBS-amplification conditions. We quantified tracking performance using the time delay between beep onset and the peak angular displacement of a movement cycle. See Fig. 4B. The dispersion of the tracking delay distribution in each condition was measured via the median absolute deviation (MAD). We assessed statistical differences in tracking delay variances between conditions (e.g., eiDBS-suppression vs. eiDBS-amplification) via the Square Rank Test(Conover and Iman, 1981). P-values were corrected for the two hypotheses (comparisons) tested by using the Bonferroni method.

UPDRS-III subscores for bradykinesia (pronation supination and upper arm/hand movements) were obtained by a clinical investigator in the off-stimulation condition two hours prior to the eiDBS experiments. We also reviewed the UPDRS-III subscores obtained eight months before surgery during the most recent clinic visit in which off-medication assessments were performed. UPDRS-III subscores were not obtained while eiDBS was delivered.

\section{Bibliography}

Aman, J.E., Johnson, L.A., Sanabria, D.E., Wang, J., Patriat, R., Hill, M., Marshall, E., MacKinnon, C.D., Cooper, S.E., Schrock, L.E., et al. (2020). Directional deep brain stimulation leads reveal spatially distinct oscillatory activity in the globus pallidus internus of Parkinson's disease patients. Neurobiol. Dis. 139, 104819.

Bevan, M.D., Magill, P.J., Terman, D., Bolam, J.P., and Wilson, C.J. (2002). Move to the rhythm: oscillations in the subthalamic nucleus-external globus pallidus network. Trends in Neurosciences 25, 525-531.

Blumenfeld, Z., Velisar, A., Miller Koop, M., Hill, B.C., Shreve, L.A., Quinn, E.J., Kilbane, C., Yu, H., Henderson, J.M., and Brontë-Stewart, H. (2015). Sixty hertz neurostimulation amplifies subthalamic neural synchrony in Parkinson's disease. PLoS ONE 10, e0121067.

Brown, P. (2003). Oscillatory nature of human basal ganglia activity: Relationship to the pathophysiology of Parkinson's disease. Mov Disord. 18, 357-363.

Brown, P., and Williams, D. (2005). Basal ganglia local field potential activity: Character and functional significance in the human. Clinical Neurophysiology 116, 2510-2519.

Brown, P., Oliviero, A., Mazzone, P., Insola, A., Tonali, P., and Di Lazzaro, V. (2001). Dopamine Dependency of Oscillations between Subthalamic Nucleus and Pallidum in Parkinson's Disease. J. Neurosci. 21, 1033-1038.

Cagnan, H., Pedrosa, D., Little, S., Pogosyan, A., Cheeran, B., Aziz, T., Green, A., Fitzgerald, J., Foltynie, T., Limousin, P., et al. (2017). Stimulating at the right time: phase-specific deep brain stimulation. Brain 140, 132-145.

Chen, C., Litvak, V., Gilbertson, T., Kuhn, A., Lu, C., Lee, S., Tsai, C., Tisch, S., Limousin, P., and Hariz, M. (2007). Excessive synchronization of basal ganglia neurons at $20 \mathrm{~Hz}$ slows movement in Parkinson's disease. Experimental Neurology 205, 214-221. 
Connolly, A.T., Jensen, A.L., Baker, K.B., Vitek, J.L., and Johnson, M.D. (2015). Classification of pallidal oscillations with increasing parkinsonian severity. J Neurophysiol 114, 209-218.

Conover, W.J., and Iman, R.L. (1981). Rank Transformations as a Bridge Between Parametric and Nonparametric Statistics. The American Statistician 35, 124-129.

DeLong, M.R. (1971). Activity of pallidal neurons during movement. Journal of Neurophysiology 34, 414-427.

Elder, C.M., Hashimoto, T., Zhang, J., and Vitek, J.L. (2005). Chronic implantation of deep brain stimulation leads in animal models of neurological disorders. Journal of Neuroscience Methods 142, $11-16$.

Escobar Sanabria, D., Johnson, L.A., Nebeck, S.D., Zhang, J., Johnson, M.D., Baker, K.B., Molnar, G.F., and Vitek, J.L. (2017). Parkinsonism and Vigilance: Alteration in neural oscillatory activity and phase-amplitude coupling in the basal ganglia and motor cortex. J. Neurophysiol. jn.00388.2017.

Escobar Sanabria, D., Johnson, L.A., Yu, Y., Busby, Z., Nebeck, S., Zhang, J., Harel, N., Johnson, M.D., Molnar, G.F., and Vitek, J.L. (2020). Real-time suppression and amplification of frequencyspecific neural activity using stimulation evoked oscillations. Brain Stimulation In Press,

2020.02.09.940643.

Eusebio, A., Chen, C.C., Lu, C.S., Lee, S.T., Tsai, C.H., Limousin, P., Hariz, M., and Brown, P. (2008). Effects of low-frequency stimulation of the subthalamic nucleus on movement in Parkinson's disease. Experimental Neurology 209, 125-130.

Eusebio, A., Pogosyan, A., Wang, S., Averbeck, B., Gaynor, L.D., Cantiniaux, S., Witjas, T., Limousin, P., Azulay, J.-P., and Brown, P. (2009). Resonance in subthalamo-cortical circuits in Parkinson's disease. Brain 132, 2139-2150.

Fogelson, N., Kühn, A.A., Silberstein, P., Limousin, P.D., Hariz, M., Trottenberg, T., Kupsch, A., and Brown, P. (2005). Frequency dependent effects of subthalamic nucleus stimulation in Parkinson's disease. Neuroscience Letters 382, 5-9.

Georgopoulos, A.P., DeLong, M.R., and Crutcher, M.D. (1983). Relations between parameters of steptracking movements and single cell discharge in the globus pallidus and subthalamic nucleus of the behaving monkey. J. Neurosci. 3, 1586-1598.

Griffiths, D.J. (2017). Electrostatics. In Introduction to Electrodynamics, (Cambridge: Cambridge University Press), pp. 59-112.

Guemas, V., Auger, L., and Doblas-Reyes, F.J. (2014). Hypothesis Testing for Autocorrelated Short Climate Time Series. J. Appl. Meteor. Climatol. 53, 637-651.

Hellerbach, A., Dembek, T.A., Hoevels, M., Holz, J.A., Gierich, A., Luyken, K., Barbe, M.T., Wirths, J., Visser-Vandewalle, V., and Treuer, H. (2018). DiODe: Directional Orientation Detection of Segmented Deep Brain Stimulation Leads: A Sequential Algorithm Based on CT Imaging. Stereotact Funct Neurosurg 96, 335-341.

Hentschke, H., and Stüttgen, M.C. (2011). Computation of measures of effect size for neuroscience data sets. European Journal of Neuroscience 34, 1887-1894. 
Holt, A.B., Wilson, D., Shinn, M., Moehlis, J., and Netoff, T.I. (2016). Phasic Burst Stimulation: A Closed-Loop Approach to Tuning Deep Brain Stimulation Parameters for Parkinson's Disease. PLoS Comput Biol 12.

Holt, A.B., Kormann, E., Gulberti, A., Pötter-Nerger, M., McNamara, C.G., Cagnan, H., Baaske, M.K., Little, S., Köppen, J.A., Buhmann, C., et al. (2019). Phase-Dependent Suppression of Beta Oscillations in Parkinson's Disease Patients. J. Neurosci. 39, 1119-1134.

Huang, H., Watts, R.L., and Montgomery, E.B. (2014). Effects of deep brain stimulation frequency on bradykinesia of Parkinson's disease. Movement Disorders 29, 203-206.

Johnson, M.D., and Mclntyre, C.C. (2008). Quantifying the Neural Elements Activated and Inhibited by Globus Pallidus Deep Brain Stimulation. Journal of Neurophysiology 100, 2549-2563.

Johnson, L.A., Aman, J.E., Yu, Y., Sanabria, D.E., Wang, J., Hill, M., Dharnipragada, R., Patriat, R., Fiecas, M., Li, L., et al. High-Frequency Oscillations in the Pallidum: A Pathophysiological Biomarker in Parkinson's Disease? Movement Disorders n/a.

Kühn, A.A., Kupsch, A., Schneider, G.-H., and Brown, P. (2006). Reduction in subthalamic 8-35 Hz oscillatory activity correlates with clinical improvement in Parkinson's disease. Eur. J. Neurosci. 23, 1956-1960.

Kühn, A.A., Tsui, A., Aziz, T., Ray, N., Brücke, C., Kupsch, A., Schneider, G.-H., and Brown, P. (2009). Pathological synchronisation in the subthalamic nucleus of patients with Parkinson's disease relates to both bradykinesia and rigidity. Experimental Neurology 215, 380-387.

Little, S., Pogosyan, A., Kuhn, A.A., and Brown, P. (2012). Beta band stability over time correlates with Parkinsonian rigidity and bradykinesia. Experimental Neurology 236, 383-388.

Ljung, L. (1986). System Identification: Theory for the User (Upper Saddle River, NJ, USA: PrenticeHall, Inc.).

Louie, K.H., Lu, C., Abdallah, T., Guzior, J.C., Twedell, E., Netoff, T.I., and Cooper, S.E. (2021). Gait phase triggered deep brain stimulation in Parkinson's disease. Brain Stimulation: Basic, Translational, and Clinical Research in Neuromodulation 14, 420-422.

Malekmohammadi, M., Shahriari, Y., AuYong, N., O’Keeffe, A., Bordelon, Y., Hu, X., and Pouratian, N. (2018). Pallidal stimulation in Parkinson disease differentially modulates local and network $\beta$ activity. Journal of Neural Engineering 15, 056016.

Nunez, P.L., and Srinivasan, R. Electric Fields of the Brain: The neurophysics of EEG (Oxford University Press).

Peles, O., Werner-Reiss, U., Bergman, H., Israel, Z., and Vaadia, E. (2020). Phase-Specific Microstimulation Differentially Modulates Beta Oscillations and Affects Behavior. Cell Reports 30, 25552566.e3.

Tass, P.A. (2002). Effective desynchronization with bipolar double-pulse stimulation. Phys Rev E Stat Nonlin Soft Matter Phys 66, 036226. 
Vitek, J.L., Bakay, R.A.E., Hashimoto, T., Kaneoke, Y., Mewes, K., Zhang, J.Y., Rye, D., Starr, P., Baron, M., Turner, R., et al. (1998). Microelectrode-guided pallidotomy: technical approach and its application in medically intractable Parkinson's disease. Journal of Neurosurgery 88, 1027-1043.

Wang, D.D., Hemptinne, C. de, Miocinovic, S., Ostrem, J.L., Galifianakis, N.B., Luciano, M.S., and Starr, P.A. (2018). Pallidal Deep-Brain Stimulation Disrupts Pallidal Beta Oscillations and Coherence with Primary Motor Cortex in Parkinson's Disease. J. Neurosci. 38, 4556-4568.

Wilson, C.J., and Bevan, M.D. (2011). Intrinsic dynamics and synaptic inputs control the activity patterns of subthalamic nucleus neurons in health and in Parkinson's disease. Neuroscience 198, 5468.

Zanos, S., Rembado, I., Chen, D., and Fetz, E.E. (2018). Phase-Locked Stimulation during Cortical Beta Oscillations Produces Bidirectional Synaptic Plasticity in Awake Monkeys. Current Biology 28, 2515-2526.e4.

(2011). Serial Correlation. In The Statistical Analysis of Time Series, (John Wiley \& Sons, Ltd), pp. 254-370. 
A Schematic of eiDBS

Amplifier

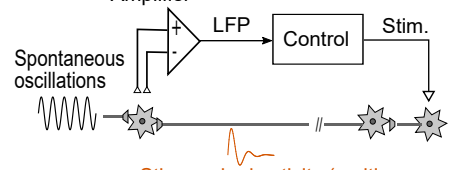

Stim-evoked activity (multi-

synaptic and/or collateral)

GPi PSD

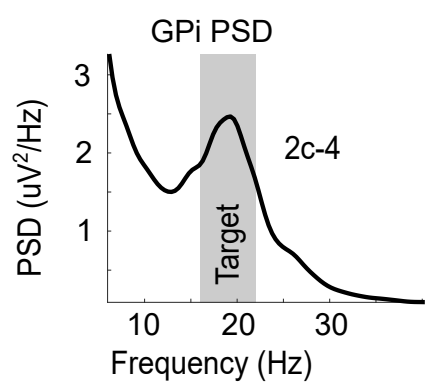

Frequency $(\mathrm{Hz})$
B

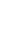

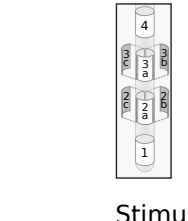

D GPi Evoked Response (ER)

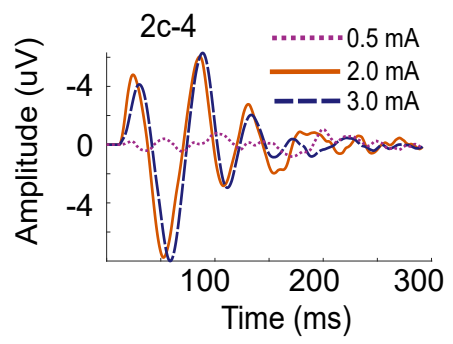

F Correlation ER and Spontaneous LFP in $16-22 \mathrm{~Hz}$ Band

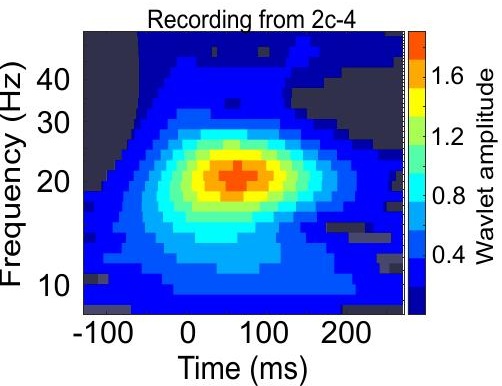

Stimulation: 3abc Recording: 2c-4

DBS Lead Topography

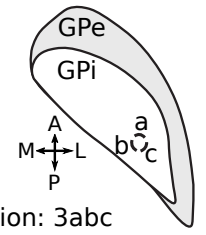

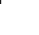

GPi ER Scalogram

Recording from 2c-4

$E$

Time (ms)

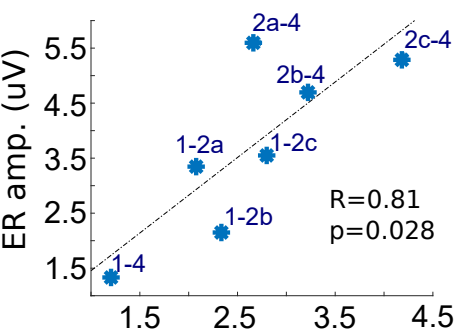

Spontaneous LFP amp. (uV) 


\section{Schematic of eiDBS Simulation}

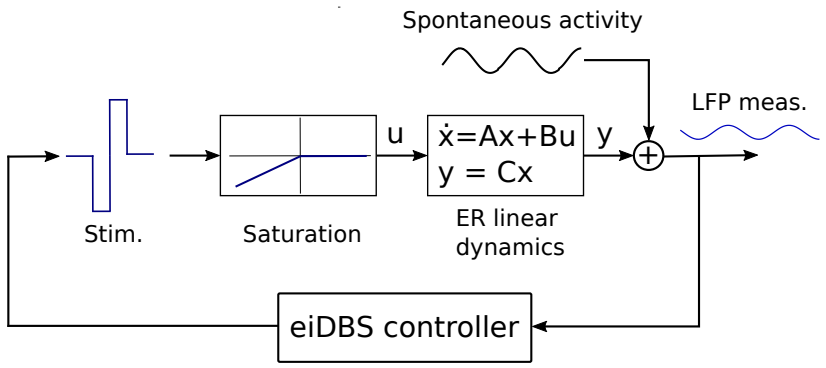

\section{ER Mathematical Model}

C Computer Optimization
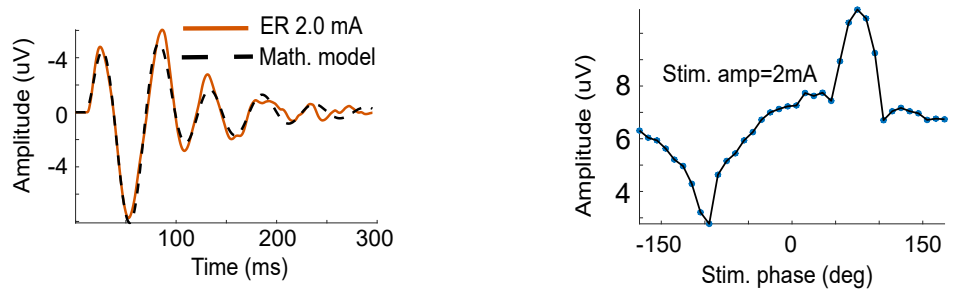

Computer Simulation of eiDBS Experiment Off Sup. Amp.

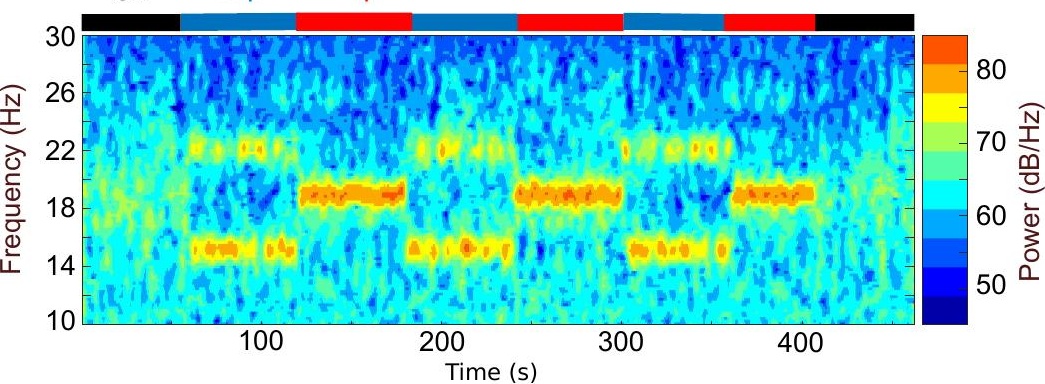


